\title{
Evaluation of Microstructural Factors Affecting the Mechanical Properties of Thin-Wall Ductile Iron Castings
}

\author{
A. Javaid and K.G. Davis
}

\author{
Materials Technology Laboratory-CANMET, 568 Booth St., Ottawa, ON, K1A 0G1, Canada
}

The automotive industry is increasingly looking at stronger and lightweight materials for use in engine production in order to meet forthcoming emissions regulations and increased market competition. In particular, there has been an increasing demand over recent years for thin-wall $(<3 \mathrm{~mm})$ ductile iron castings to provide components with high strength-to-weight ratios. Reducing the weight of ductile iron castings by producing thin-wall parts is an important method for saving energy and materials, and hence reducing emissions. In production, however, the high cooling rate of thin-section ductile iron results in metallurgical problems of carbide formation, microporosity, and misruns, which adversely affect the machinability and mechanical properties. The production of thin-wall iron castings is presently limited by a lack of metallurgical processing guidelines. The technical challenge is associated both with precise metallurgical process control to produce a carbide-free structure, and with the establishment of good mold filling ability through appropriate casting techniques. The energy requirement for melting iron is much lower than for other alloys, as confirmed by recent energy audits in several Canadian foundries using the Mobile Foundry Laboratory. Therefore, developing and perfecting thin-wall iron casting technology will not only result in weight reduction of the components presently made of iron, but will also provide a viable alternative material for some components that is cheaper and results in less $\mathrm{CO}_{2}$ emissions from the utilities point of view. The transfer of thin-wall iron casting technology to Canadian automotive parts producers will increase their competitiveness and technical capability to respond to increasing demands for lightweight materials that will lead to lower vehicle weights and reduced emissions.

The objective of this research is to develop and advance thin-wall iron casting technology to produce strong, lightweight iron castings at lower cost for automotive applications. In preliminary work done at MTL-CANMET, carbide-free thin-wall (down to $1 \mathrm{~mm}$ ) ductile iron castings with good strength properties were produced by optimizing the chemical composition, post inoculation procedures, and melt pre-conditioning, along with precise control of the melting process and the use of high purity charge materials. Carbide formation was decreased by the use of moldable insulating materials, certain molding systems/binders, and a finer sand grain size. This research has resulted in 7 presentations and 5 publications in refereed journals, and has led to a task-shared project on thin-wall ductile iron castings with Rio Tinto where the mold filling characteristics will be examined using vacuum assisted casting methods.

The effect of microstructure achieved under various molten metal processing conditions and melt chemistries on the mechanical properties was investigated. Microstructure evaluation was done (close to the fracture end of test samples) to characterize the graphite count, shape, size, and distribution in the unetched condition and ferrite, pearlite and carbide content were determined by etching with $2 \%$ nital and $10 \%$ ammonium persulfate solution, respectively. The standard procedures for microstructure evaluation (i.e., comparison method at 100X) cannot be used to compare such an extremely high nodule count in thin sections (e.g., 5000 nodules per $\mathrm{mm}^{2}$ in $1 \mathrm{~mm}$ section) and needs to be modified. Therefore, the nodule count was determined by counting all the nodules at 500X and reporting them for 1 $\mathrm{mm}^{2}$ area. A detailed assessment of the graphite structure of some selected samples was made by using image analysis. A total of 12 representative images were taken from all the areas of each sample and averaged to report the microstructure. Fig 1 shows a comparison of results from the two methods. They are very close, with counts from the image analyser being slightly higher than those obtained with the manual method. Fig. 2 shows the nodule size distribution from image analyzer $\left(1 \mathrm{~mm}^{2}\right.$ area) in $3,2.5,2,1.5$, and $1 \mathrm{~mm}$ sections. It was observed that more than $50 \%$ of nodules present in thin sections $(<3 \mathrm{~mm})$ were less than 5 micron in size. The average nodule size ranges from 3.92$5.88 \mu \mathrm{m}$ for $1 \mathrm{~mm}, 5.02-6.81 \mu \mathrm{m}$ for $2 \mathrm{~mm}$, and $5.25-7.30 \mu \mathrm{m}$ for $3 \mathrm{~mm}$ sections. Figs. 3 and 4 show the overall effect of different section sizes on average nodule count, carbides, and ferrite content.

Microstructures and mechanical properties in 3 and $12 \mathrm{~mm}$ sections within the same casting were compared. Some examples of microstructures at fracture surfaces are shown in Figs. 5 through 8. While many samples from 3-mm sections showed low elongation values, caused by a high pearlite content and/or the presence of carbides, many others showed high elongations and superior strengths. At moderate to high elongations, the 3-mm section samples were significantly stronger as a result of more refined and homogeneous microstructure. In the 12-mm sections, the microstructures had a controlling influence on the tensile and impact properties. In 3-mm sections, while the tensile properties were also strongly dependent on microstructure, the impact properties were relatively insensitive. The most significant microstructural factor affecting the tensile properties in both section sizes was the ferrite content. Of the elements contained in the iron, silicon had the greatest effect on the microstructure in promoting ferrite and improving the tensile properties for both sections. Despite the promotion of ferrite by silicon, the Charpy toughness decreased with increased silicon in the $12 \mathrm{~mm}$ sections, and was essentially independent of silicon content in the $3 \mathrm{~mm}$ sections. 

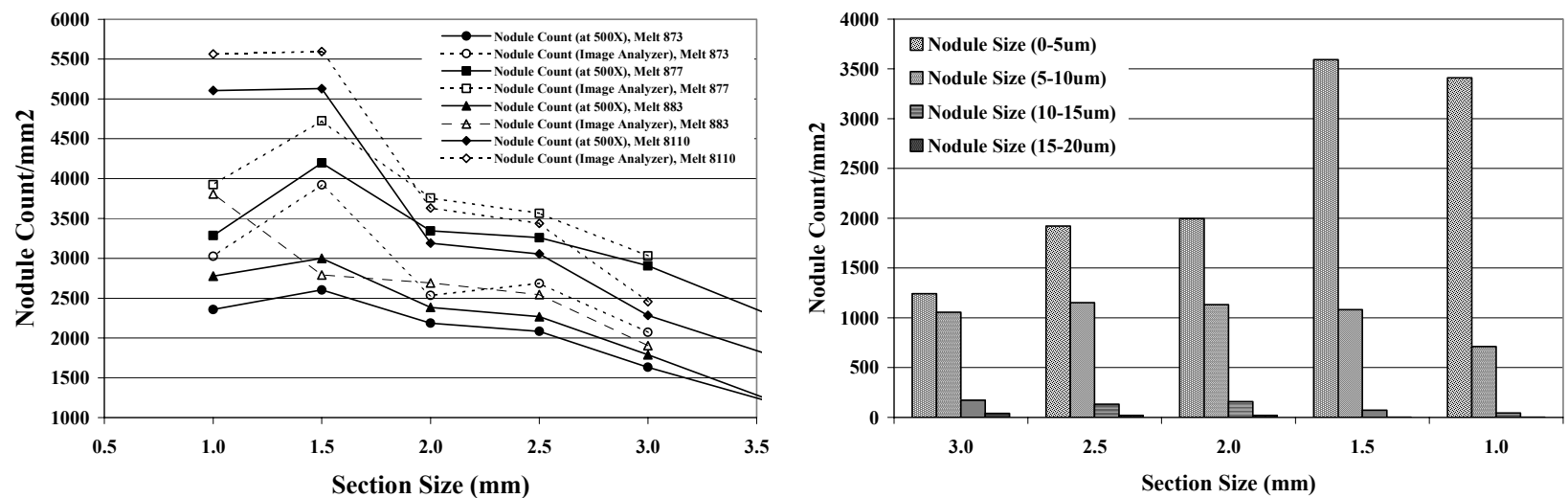

Figs. 1 and 2. Comparison of nodule counts from image analyzer \& at 500X magnification for $1 \mathrm{~mm}^{2}$ area (left) and nodule size distribution from image analyzer $\left(1 \mathrm{~mm}^{2}\right.$ area) in $3,2.5,2,1.5$, and $1 \mathrm{~mm}$ sections (right).
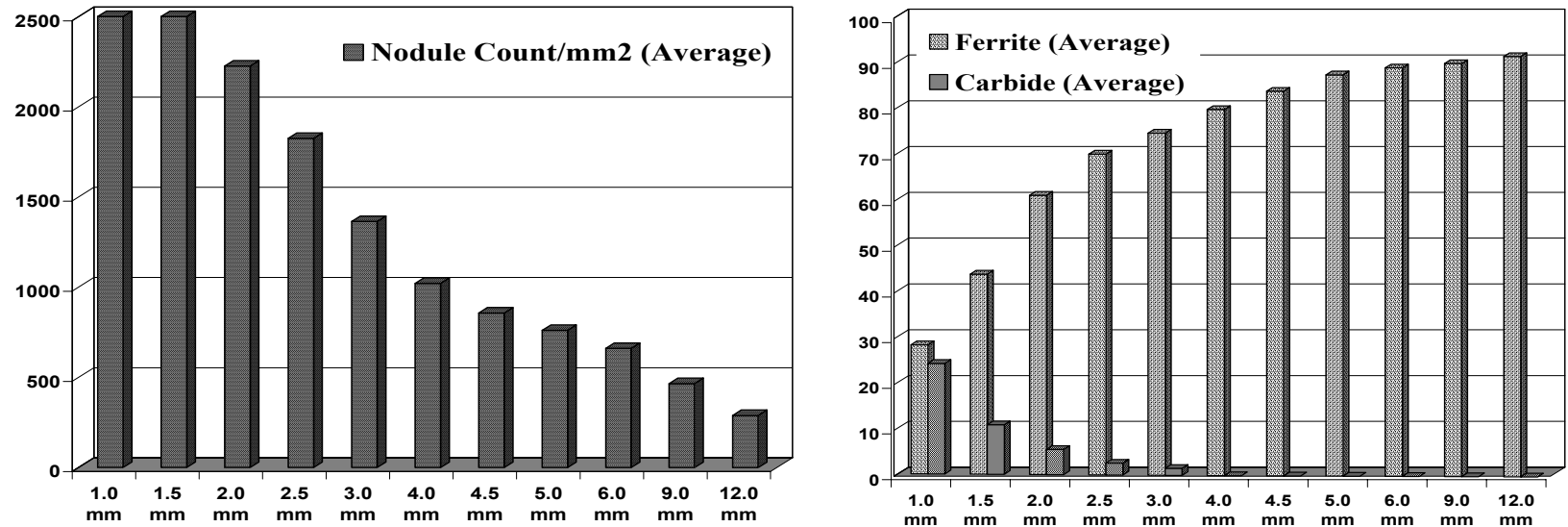

Figs. 3 and 4. Effect of section size on average nodule count (left) and average carbide and ferrite contents (right).
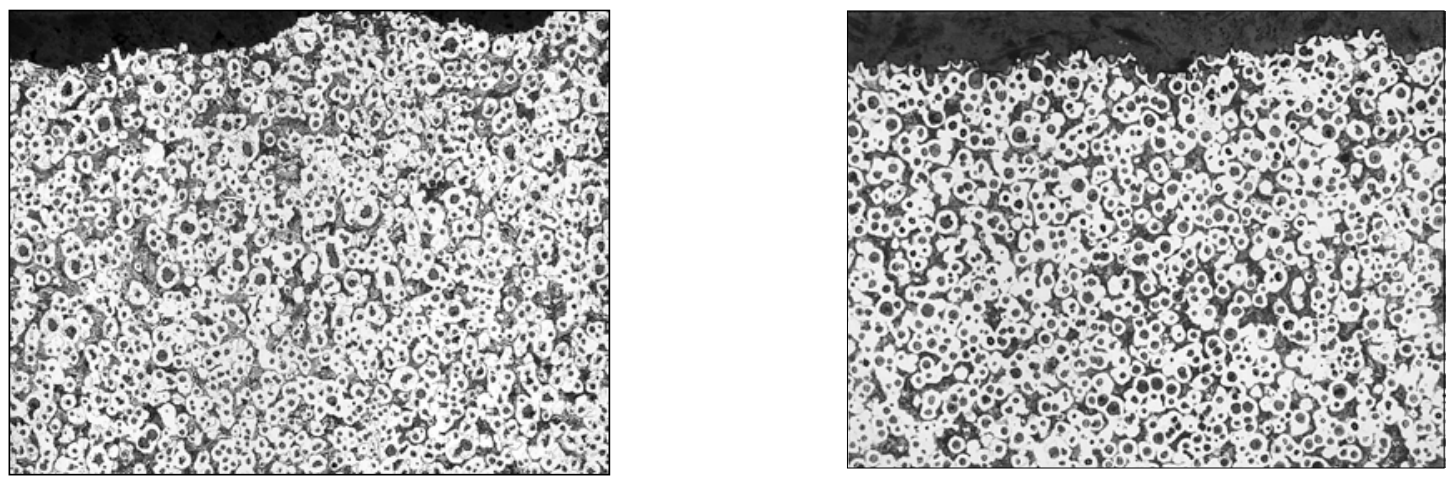

Figs. $5 \&$ 6. Microstructure from fractured end of 12 (left) \& $3 \mathrm{~mm}$ (right) tensile samples within the same casting100X.
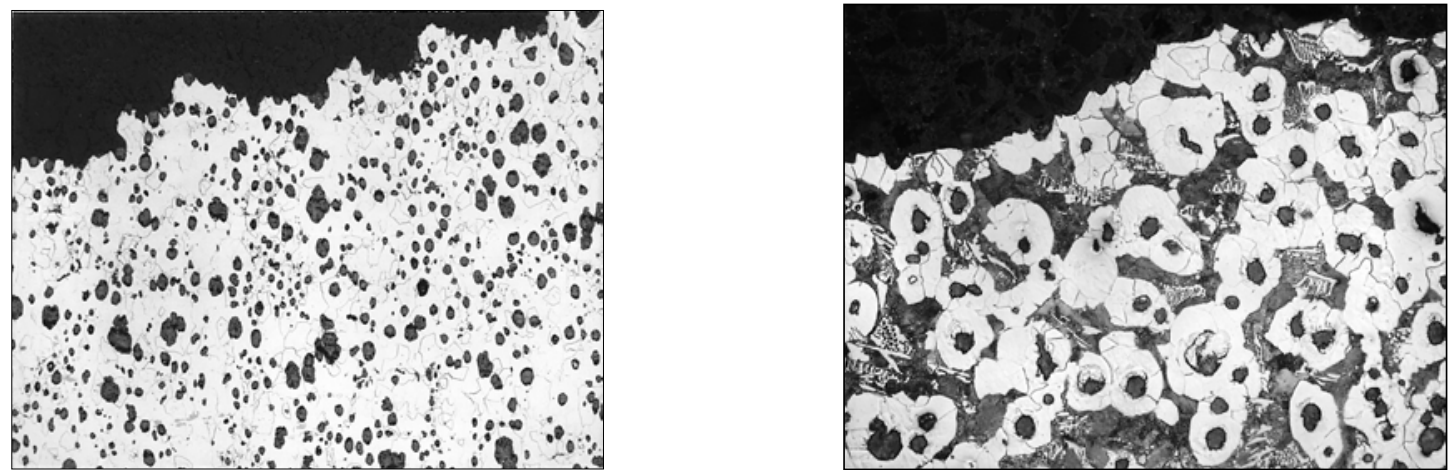

Figs. $7 \&$ 8. Microstructure from fractured end of $3 \mathrm{~mm}$ tensile sample, 100X (left) \& presence of carbides, $500 \mathrm{X}$ (right) 